\title{
Contribuição ao estudo da estabilidade de edifícios de andares múltiplos em aço
}

\author{
Rafael Eclache Moreira de Camargo ${ }^{1 *}$ e José Jairo de Sáles² \\ ${ }^{1}$ Mestre em Engenharia de Estruturas, EESC-USP, \\ eclachecamargo@yahoo.com.br \\ 2 Professor aposentado do Departamento de Engenharia de Estruturas da EESC- \\ USP, jjsales@sc.usp.br
}

\section{Contribution to the study of stability of steel multi-storey buildings}

\begin{abstract}
Resumo
Este trabalho apresenta uma análise comparativa de diferentes sistemas estruturais de um edifício de 20 pavimentos com o objetivo de avaliar a influência da concepção estrutural nos efeitos de segunda ordem. Cada um dos modelos foi dimensionado pelos princípios do método da análise direta, presente na ABNT NBR 8800:2008. O método da amplificação dos esforços solicitantes (MAES) foi usado para se obter os esforços atuantes nos elementos dos edifícios considerando os efeitos locais e globais de segunda ordem. A incidência do vento foi simulada sem e com excentricidade devida aos efeitos de vizinhança, de acordo com a ABNT NBR 6123:1988. Todas as análises numéricas foram repetidas usando o método simplificado de segunda ordem conhecido como P-Delta. Os resultados obtidos foram comparados com o objetivo de se obter diretrizes de ordem prática para o dimensionamento de edifícios em aço.
\end{abstract}

Palavras-chave: Edifícios em aço. Sistemas estruturais. Estabilidade estrutural. Análise de segunda ordem. Efeitos de vizinhança.

\begin{abstract}
This work presents a comparative analysis of different structural systems of a 20-storey building with the objective of evaluating the influence of structural design on second-order effects. Each model has been designed using the principles of the direct analysis method (DAM), present in the ABNT NBR 8800:2008. The first-order amplification method (FOAM) was used to obtain the forces acting on the building elements, including the local and global second-order effects. The incidence of the wind was simulated without and with an eccentricity due to the vicinity effects, according to ABNT NBR 6123:1988. All numerical analyses were repeated using the second-order simplified method known as P-Delta. The results were compared in order to obtain guidelines for the design of steel multi-storey buildings.
\end{abstract}

Keywords: Steel buildings. Structural systems. Structural stability. Second-order analysis. Vicinity effects.

\footnotetext{
* autor correspondente
} 


\section{Introdução}

O crescimento demográfico presenciado nas últimas décadas tornou escassa a disponibilidade de grandes terrenos nas áreas urbanas, gerando uma verticalização dos empreendimentos. Por questões culturais, o material que teve maior aceitação no Brasil foi o concreto armado. Entretanto, nos últimos anos houve um crescimento na utilização de sistemas estruturais mistos em aço e concreto, devido a algumas vantagens quando comparados com os sistemas tradicionais, como a diminuição das seções transversais, a redução dos custos com fôrmas e escoramentos, a maior organização dos canteiros de obra e a redução dos prazos de execução.

O comportamento dessas estruturas não é simples e por essa razão algumas simplificações são necessárias para viabilizar o seu estudo. Um tipo de simplificação bastante comum nas análises estruturais diz respeito à consideração da ação do vento nos edifícios. Geralmente, os carregamentos devidos ao vento são considerados uniformes (sem excentricidades), o que muitas vezes não ocorre devido à presença de outras construções próximas, responsáveis por gerar os efeitos de vizinhança.

Além disso, também é de fundamental importância a consideração do seu comportamento global para que os esforços sejam adequadamente mensurados. Segundo Ziemian (2010), o requisito básico para uma análise de segunda ordem é assegurar que o equilíbrio da estrutura seja satisfeito em sua condição deformada. Devido à sua complexidade, é comum a utilização de métodos simplificados para se levar em conta os efeitos de segunda ordem, inserindo tanto as imperfeições de material quanto a não linearidade geométrica na análise estrutural.

\subsection{Objetivos}

O presente trabalho tem como objetivo principal realizar a análise numérica de edifícios de andares múltiplos em aço para avaliar a influência da concepção estrutural nos efeitos de segunda ordem. Além disso, pretende-se estudar a influência dos efeitos de vizinhança na estabilidade dos edifícios, avaliar a aplicabilidade do método simplificado de análise de segunda ordem proposto pela ABNT NBR 8800:2008 e comparar os valores obtidos por essa metodologia com os resultados fornecidos por software comercial empregando o método P-Delta. 


\subsection{Justificativas}

A publicação da ABNT NBR 8800:2008 trouxe algumas modificações significativas. Na sua nova versão, a norma brasileira de aço passou a exigir a consideração dos efeitos de segunda ordem na determinação dos esforços. Por essa razão, existe a necessidade de serem publicados trabalhos que abordem os novos conceitos introduzidos e mostrem por meio de exemplos práticos a sua aplicação.

Analisando a literatura existente, observa-se que a maioria dos trabalhos que abordam a estabilidade global de estruturas metálicas foi realizada por meio da análise de pórticos planos. Esse tipo de abordagem tem como vantagem a simplificação do modelo matemático e facilidade na interpretação dos resultados. No entanto, ela não possibilita a avaliação dos efeitos de torção na estrutura e pode não simular de um modo realístico o seu comportamento.

Além disso, apesar de fazerem parte da ABNT NBR 6123:1988, os efeitos de vizinhança muitas vezes não são considerados no dimensionamento de estruturas. Dessa maneira, pouco se sabe sobre seus efeitos na estabilidade de edifícios.

\section{Metodologia}

Para o desenvolvimento deste trabalho foram realizadas análises numéricas de três diferentes edifícios por meio do software SAP2000. No sentido de avaliar os modelos numéricos elaborados, os resultados obtidos pelo método simplificado da ABNT NBR 8800:2008 foram comparados com os valores fornecidos por análises que consideram a não linearidade geométrica pelo método P-Delta. Um estudo sobre a influência dos efeitos de vizinhança na estabilidade dos edifícios foi realizado em seguida, confrontando os resultados das análises que apresentam esses efeitos com os valores obtidos em modelagens que os desconsideram, aqui chamadas de "vento uniforme".

\section{Desenvolvimento}

\subsection{Descrição geral da estrutura}

Os edifícios analisados neste trabalho possuem as mesmas características arquitetônicas, mas diferenciam-se pelo sistema estrutural adotado. A estrutura utilizada como base para os modelos foi retirada de Sáles (1995) e consiste num 
edifício comercial de 20 pavimentos, com pé-direito único, igual a 3,5 $\mathrm{m}$. Suas dimensões em planta são $45 \times 20 \mathrm{~m}$, totalizando $18.000 \mathrm{~m}^{2}$, ou $900 \mathrm{~m}^{2}$ por andar. Em todos os casos analisados foram utilizados pilares metálicos, vigas mistas em aço e concreto, e lajes do tipo steel deck. A construção foi considerada não escorada.

As filas e os eixos da edificação são mostrados na Figura 1. Na direção de maior comprimento foram utilizados 5 vãos de $9 \mathrm{~m}$, enquanto que na outra direção foram empregados nas extremidades dois vãos de $8 \mathrm{~m}$ e um vão central de $4 \mathrm{~m}$. Considerouse que entre as filas B e $C$ existam duas regiões destinadas a escadas e elevadores.

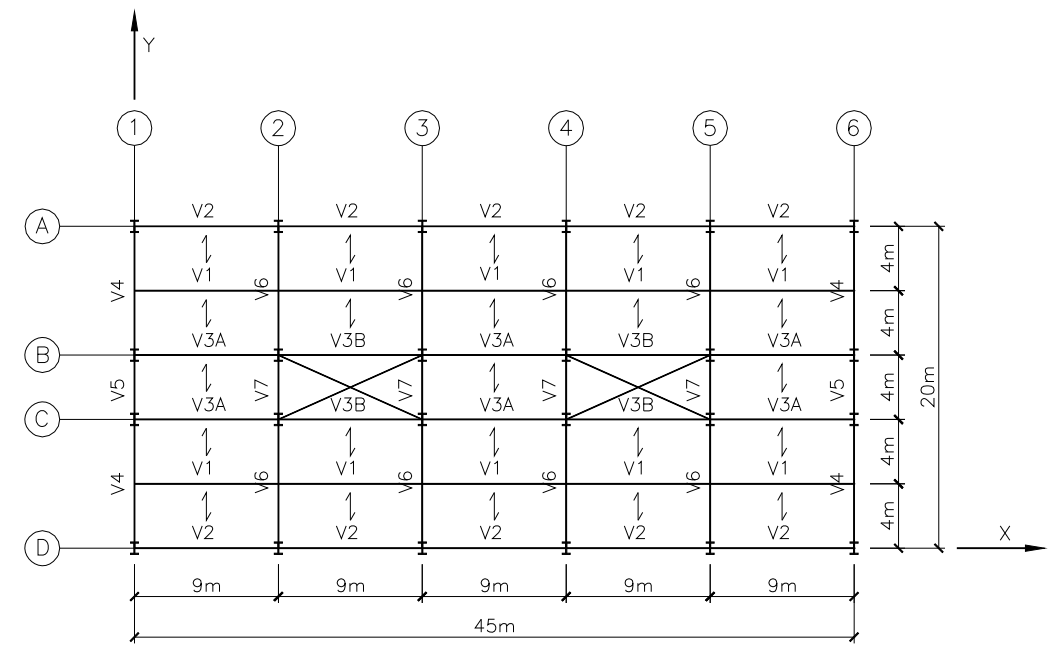

Figura 1 - Pavimento tipo do edifício modelo. (Fonte: Baseado em Sáles, 1995)

Os resultados apresentados a seguir são referentes aos modelos 1,2 e 3 . O modelo 1 trata-se de um edifício todo aporticado. O modelo 2 difere do primeiro modelo pela inclusão de contraventamentos em X nos eixos 1 e 6 , e em $K$, nas filas $A$ e D. Por sua vez, o modelo 3 difere do modelo 2 pela inclusão de contraventamentos em $X$ nos eixos 2 e 5. Outras informações estão apresentadas nos itens 4.1, 4.2 e 4.3.

\subsection{Características dos materiais}

Para as vigas mistas e pilares metálicos foram utilizados aços USI CIVIL 350. Os contraventamentos, quando empregados, foram dimensionados com aço A572 Gr.50. Nas vigas e lajes mistas foi considerado concreto com $f_{c k}$ igual a $20 \mathrm{MPa}$. O steel deck utilizado nos modelos foi o MF-75, de aço galvanizado ASTM A653 Grau 40 (ZAR-280), com 1,25 mm de espessura. A altura total da laje adotada foi de $150 \mathrm{~mm}$. As armaduras longitudinais utilizadas foram do tipo CA-50. 


\subsection{Levantamento das ações}

As ações permanentes consideradas nos pisos foram: peso próprio da laje (2,79 $\left.\mathrm{kN} / \mathrm{m}^{2}\right)$; divisórias $\left(1,0 \mathrm{kN} / \mathrm{m}^{2}\right)$; forro e serviços $\left(0,5 \mathrm{kN} / \mathrm{m}^{2}\right)$; revestimento $\left(0,65 \mathrm{kN} / \mathrm{m}^{2}\right)$ e pele de vidro $(1,25 \mathrm{kN} / \mathrm{m})$. No último pavimento (cobertura) as ações permanentes adotadas foram: peso próprio da laje $\left(2,79 \mathrm{kN} / \mathrm{m}^{2}\right)$; forro e serviços $\left(0,5 \mathrm{kN} / \mathrm{m}^{2}\right)$; impermeabilização $\left(0,75 \mathrm{kN} / \mathrm{m}^{2}\right)$ e pele de vidro $(1,25 \mathrm{kN} / \mathrm{m})$. Os pesos próprios das vigas e pilares foram aplicados diretamente nas barras dos modelos de acordo com seus valores lineares $(\mathrm{kN} / \mathrm{m})$.

Também foi considerada no topo dos edifícios a existência de cargas permanentes devidas a dois reservatórios de água. Esse carregamento foi aplicado diretamente nos pilares posicionados nos painéis onde os reservatórios foram locados, entre os eixos 3 e 4, totalizando $245 \mathrm{kN}$ em cada pilar.

As sobrecargas de utilização e de construção assumidas nos modelos foram de 2,0 $\mathrm{kN} / \mathrm{m}^{2}$ e $1,0 \mathrm{kN} / \mathrm{m}^{2}$, respectivamente, conforme ABNT NBR 6120:1980 e o anexo B da ABNT NBR 8800:2008.

O carregamento lateral devido ao vento foi estabelecido conforme a ABNT NBR 6123:1988, e aplicado diretamente nos pilares por meio de carregamentos distribuídos, considerando suas respectivas áreas de influência. Por simplificação, considerou-se a atuação do vento apenas na direção de menor inércia do edifício ( $Y$ ) para o dimensionamento dos elementos aos estados limites últimos. Nos estados limites de serviço, a ação do vento foi analisada nas duas direções. Em ambos os casos, os coeficientes adotados foram $V_{0}=40 \mathrm{~m} / \mathrm{s} ; \mathrm{S}_{1}=\mathrm{S}_{3}=1,0 ; \mathrm{S}_{2}$ - categoria IV, classe $\mathrm{C}$, variando a cada $3,5 \mathrm{~m} ; \mathrm{C}_{\mathrm{a}}=1,10$ (face maior) e 0,85 (face menor).

As forças nocionais foram aplicadas nos nós superiores dos pilares, também no sentido de menor inercia do edifício $(\mathrm{Y})$.

Para a análise dos efeitos de vizinhança foi utilizada uma excentricidade igual a $15 \%$ do comprimento das faces dos edifícios estudados. Tendo conhecimento do momento de torção atuante em cada pavimento, no topo dos pilares foram aplicadas forças concentradas formando binários entre os eixos 1 e 6, 2 e 5, 3 e 4, para o vento na direção $Y$; e filas $A$ e $D, B$ e $C$, para o vento na direção $X$. As forças de cada binário 
foram obtidas dividindo a força resultante devida ao vento no nível de cada pavimento pelo número de pórticos resistentes a esse carregamento em cada direção ( 6 na direção $Y$ e 4 na direção $X)$ e considerando que seus valores fossem diretamente proporcionais às suas distâncias em relação ao centro de gravidade dos pisos. Assim, as forças de arrasto foram sobrepostas com as forças desses binários para que a resultante de cada pavimento não se alterasse.

Uma outra alternativa para considerar esses efeitos seria a aplicação direta do momento de torção, das forças resultantes do vento e das forças nocionais no nó mestre de cada piso. Essa segunda opção, embora não tenha sido utilizada nesse trabalho, é menos trabalhosa e facilita a modelagem das edificações.

\subsection{Combinações de ações}

As combinações últimas normais foram consideradas para quatro situações diferentes, listadas abaixo com os coeficientes de ponderação e fatores de combinação adotados:

- Sobrecarga como ação variável principal:

$$
1,25 P P_{1}+1,40 P P_{2}+1,50 P P_{3}+1,40 P P_{4}+1,50 S C+(1,40 \times 0,6) V
$$

- Vento como ação variável principal:

$$
1,25 P P_{1}+1,40 P P_{2}+1,50 P P_{3}+1,40 P P_{4}+1,40 V+(1,50 \times 0,7) S C
$$

- Sobrecarga como ação variável principal, sem atuação do vento:

$$
1,25 P P_{1}+1,40 P P_{2}+1,50 P P_{3}+1,40 P P_{4}+1,50 S C+F N
$$

- Vento como ação variável principal, sem atuação de sobrecarga:

$$
1,25 P P_{1}+1,40 P P_{2}+1,50 P P_{3}+1,40 P P_{4}+1,40 V
$$

Onde $\mathrm{PP}_{1}$ é o peso próprio dos perfis de aço; $\mathrm{PP}_{2}$ é o peso próprio da laje; $\mathrm{PP}_{3}$ é o peso próprio das divisórias, forros e serviços, pele de vidro, revestimento e impermeabilização; $\mathrm{PP}_{4}$ é o peso próprio da caixa d'água; SC é a sobrecarga de utilização; V é a ação do vento e FN são as forças nocionais.

No que diz respeito aos estados limites de serviço, foram empregadas combinações quase permanentes [Eq. (5)] e raras [Eq. (6)] para verificar as flechas das vigas mistas, e 
apenas combinações raras [Eq. (7)] para a avaliação dos deslocamentos laterais e dos deslocamentos interpavimentos. Estas combinações estão apresentadas a seguir.

- Análise das flechas das vigas mistas:

$$
\begin{aligned}
& P P_{1}+P P_{2}+P P_{3}+0,4 S C \\
& P P_{3}+S C
\end{aligned}
$$

- Avaliação dos deslocamentos laterais e interpavimentos:

$$
P P_{1}+P P_{2}+P P_{3}+P P_{4}+V+0,6 S C
$$

Para o cálculo das flechas, os efeitos de longa duração (fluência e retração do concreto) foram considerados na homogeneização das seções multiplicando por 3 a razão modular entre o módulo de elasticidade do aço e do concreto.

\subsection{Recursos utilizados}

O programa utilizado nas simulações numéricas foi o SAP2000, que é baseado no método dos elementos finitos. Para a importação de dados para esse programa e o tratamento dos resultados obtidos foram desenvolvidas planilhas eletrônicas utilizando o software Excel e linguagem de programação VBA.

Por intermédio do SAP2000 também foram feitos outros tipos de análises elásticas simplificadas de segunda ordem. A imperfeição de material foi incorporada aos modelos pela redução do módulo de elasticidade dos materiais. A não linearidade geométrica foi considerada por meio do efeito P-Delta, existente no programa. Os efeitos locais de segunda ordem foram incorporados nas análises com o método PDelta pela colocação de três nós intermediários nas barras dos pilares.

Em relação ao dimensionamento dos elementos, foram desenvolvidas planilhas no programa Mathcad, de acordo com as recomendações presentes na ABNT NBR 8800:2008. As planilhas desenvolvidas, bem como a rotina utilizada na geração de arquivos de importação do SAP2000 contendo as propriedades geométricas de seções genéricas (vigas mistas) e perfis I estão disponíveis em Camargo (2012).

Nas análises de segunda ordem, foi utilizado o critério presente na ABNT NBR 8800:2008 de se dividir os coeficientes de ponderação das ações por 1,1 e multiplicar 
os resultados obtidos por esse mesmo valor. Para o dimensionamento dos pilares, foi adotada a redução da sobrecarga em quase todos os casos.

A análise dos deslocamentos interpavimentos foi feita levando em consideração os deslocamentos provocados pelas forças cortantes de acordo com o ângulo de distorção provocado pelas mesmas. Esses deslocamentos foram calculados para todos os andares, nas duas direções.

\subsection{Modelagem dos elementos}

As vigas e os pilares dos modelos analisados foram modelados por meio de elementos de barra (frame elements). As lajes foram modeladas como diafragmas rígidos, sendo, portanto, desconsiderada a flexão no seu plano. Esse comportamento pôde ser simulado pelo recurso do SAP2000 chamado constraint. Nesses casos, o constraint empregado em cada pavimento foi o rigid diaphragm, no qual os nós são ligados uns aos outros por links rígidos em um determinado plano, de modo que eles se movam juntos, como um diafragma. Esta hipótese simplificadora reduz o número de graus de liberdade a ser solucionado, o que torna mais rápida a análise estrutural dos modelos.

\section{Resultados}

\subsection{Modelo 1}

Este modelo consiste em uma solução estrutural formada por pórticos em todas as filas e eixos, com bases engastadas. A excentricidade do vento foi aplicada de modo que o momento de torção resultante atuasse, em planta, no sentido horário. Com o intuito de simplificar o dimensionamento dos pilares, não foi feita distinção entre pilares centrais e de fachada e não houve redução de sobrecarga. Entretanto, admitiuse que as seções variassem a cada quatro andares.

As vigas foram consideradas contínuas. Nas regiões de momento negativo, apenas as propriedades do perfil de aço foram consideradas para o cálculo da resistência. Nas regiões de momento positivo, as vigas foram consideradas mistas.

Após algumas iterações, foram obtidas vigas mistas e pilares de aço com as características indicadas na

Tabela 1 e na Tabela 2. 
Tabela 1 - Características das vigas mistas. (Fonte: Camargo, 2012)

\begin{tabular}{|c|c|c|c|c|c|}
\hline \multirow{2}{*}{ Viga } & \multirow{2}{*}{ Seção } & \multicolumn{4}{|c|}{ Perfil de aço } \\
\hline & & $\mathrm{d}(\mathrm{mm})$ & $b_{f}(m m)$ & $t_{f}(\mathrm{~mm})$ & $t_{w}(\mathrm{~mm})$ \\
\hline V1 & VS $500 \times 61$ & 500 & 250 & 9,5 & 6,3 \\
\hline V2 & VS $500 \times 61$ & 500 & 250 & 9,5 & 6,3 \\
\hline V3A & VS $500 \times 73$ & 500 & 250 & 12,5 & 6,3 \\
\hline V3B & VS $500 \times 73$ & 500 & 250 & 12,5 & 6,3 \\
\hline V4 & VS $750 \times 108$ & 750 & 320 & 12,5 & 8,0 \\
\hline V5 & VS $750 \times 108$ & 750 & 320 & 12,5 & 8,0 \\
\hline V6 & VS $750 \times 108$ & 750 & 320 & 12,5 & 8,0 \\
\hline V7 & VS $750 \times 108$ & 750 & 320 & 12,5 & 8,0 \\
\hline
\end{tabular}

Tabela 2 - Características dos pilares de aço. (Fonte: Camargo, 2012)

\begin{tabular}{ccccccc}
\hline \multirow{2}{*}{ Pilar } & \multirow{2}{*}{ Andares } & \multirow{2}{*}{ Seção } & $\mathbf{d}(\mathbf{m m})$ & $\mathbf{b}_{\mathbf{f}}(\mathbf{m m})$ & $\mathbf{t}_{\mathbf{f}}(\mathbf{m m})$ & $\mathbf{t}_{\mathbf{w}}(\mathbf{m m})$ \\
\hline P1 & $1^{\circ}-4^{\circ}$ & PS $900 \times 648$ & 900 & 700 & 44,5 & 25 \\
P2 & $5^{\circ}-8^{\circ}$ & PS $800 \times 407$ & 800 & 600 & 31,5 & 19 \\
P3 & $9^{\circ}-12^{\circ}$ & PS $720 \times 300$ & 720 & 550 & 25 & 16 \\
P4 & $13^{\circ}-16^{\circ}$ & PS $600 \times 246$ & 600 & 450 & 25 & 16 \\
P5 & $17^{\circ}-20^{\circ}$ & PS 550 5169 & 400 & 350 & 19 & 16 \\
\hline
\end{tabular}

Durante a classificação da estrutura, o máximo valor encontrado para o coeficiente $B_{2}$ foi 1,11 , obtido na combinação sem o vento e apenas com forças nocionais. Quando utilizada a relação entre os deslocamentos de segunda e primeira ordem $\left(u_{2} / u_{1}\right), 0$ maior valor encontrado foi 1,07 , para a combinação em que a sobrecarga é a ação variável principal, considerando os efeitos de vizinhança. Todos os valores do $B_{2}$ foram obtidos pelos deslocamentos dos nós superiores dos pilares, com fator $R_{s}$ igual a 0,85 .

Esse comportamento era esperado, pois sabe-se que os maiores valores do coeficiente $B_{2}$ ocorrem nas combinações com os maiores valores de cargas gravitacionais.

Assim, devido ao fato do edifício ser classificado como de média deslocabilidade pelo coeficiente $B_{2}$, deu-se prosseguimento ao seu dimensionamento com o módulo de elasticidade reduzido para simular as imperfeições iniciais de material. Nesse caso, os coeficientes $B_{1}$ ficaram limitados ao seu valor mínimo $(1,00)$ para as duas hipóteses de incidência do vento e o máximo valor de $B_{2}$ passou para cerca de 1,13 , no pórtico do eixo 3.

Na Figura 2 estão apresentados os valores de $B_{2}$ obtidos para o eixo 3, para cada uma das combinações últimas normais definidas no item 3.4, sem redução da sobrecarga e considerando o vento atuando na direção $Y$ com efeitos de vizinhança, quando aplicável. 


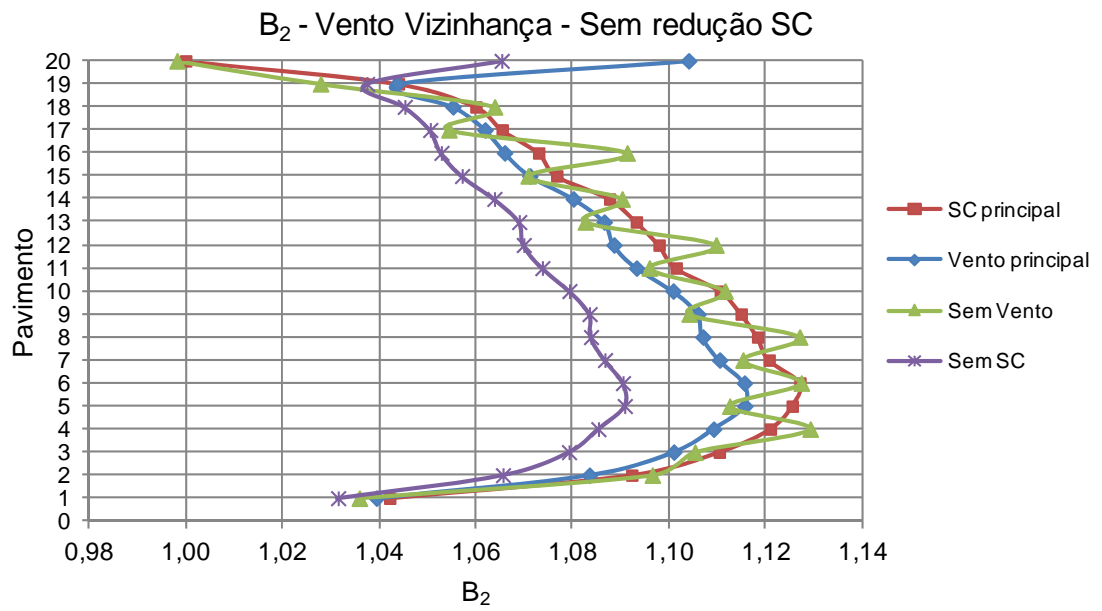

Figura 2 - Variação do coeficiente $B_{2}$, por pavimento, considerando o vento com efeitos de vizinhança em $Y$, quando aplicável, e sem redução da sobrecarga - Eixo 3.

(Fonte: Camargo, 2012)

Na Figura 3 estão indicados os valores de $B_{2}$ obtidos para o eixo 3, quando a incidência do vento é uniforme. Analisando esses resultados, percebe-se que o maior valor de $B_{2}$ ocorreu na altura do $4^{\circ}$ pavimento. Além disso, nota-se que o comportamento das curvas foi praticamente o mesmo para as duas hipóteses de incidência do vento.

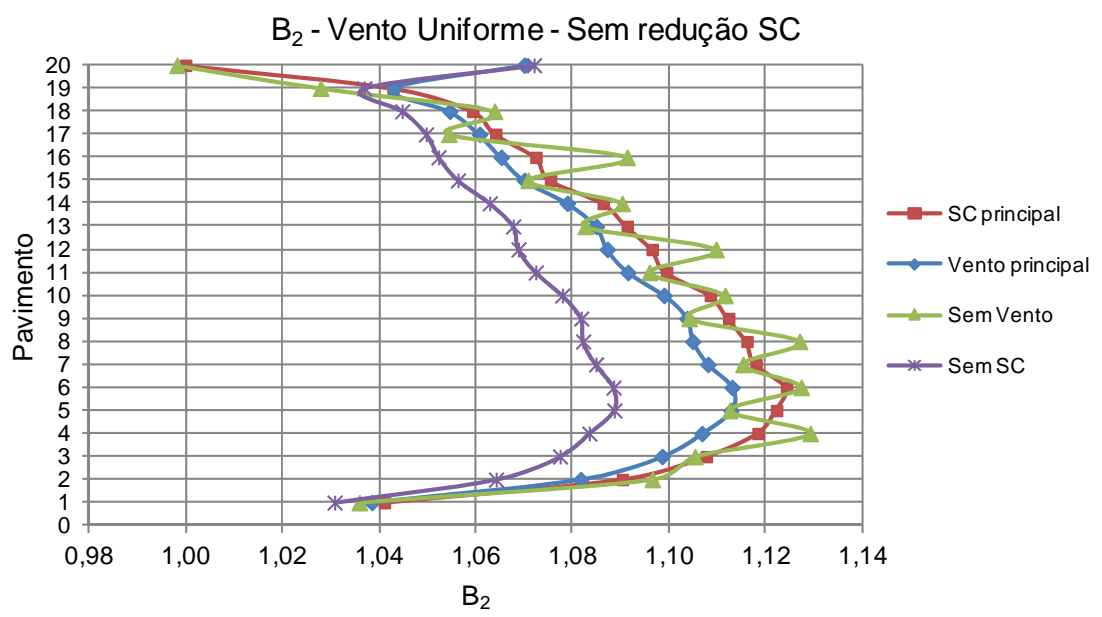

Figura 3 - Variação do coeficiente $B_{2}$, por pavimento, considerando o vento uniforme em Y, quando aplicável, e sem redução da sobrecarga - Eixo 3. (Fonte: Camargo, 2012) Comparativamente, os esforços encontrados para o vento uniforme ficaram próximos aos obtidos para o vento com os efeitos de vizinhança. Analisando os resultados das relações entre os esforços solicitantes e as resistências de cada seção, observa-se que as maiores diferenças ocorreram nos momentos fletores devido ao acréscimo de deslocamentos provenientes dos efeitos de torção. Como pode ser visto na Tabela 3, 
os esforços de flexão em relação a X (eixo de maior inércia) ficaram, em média, 5,4\% maiores, com um desvio-padrão de $5 \%$. Para os momentos fletores atuantes em $\mathrm{Y}$, os esforços com excentricidade do vento resultaram, na média, 32,7\% maiores do que os sem excentricidade. O desvio-padrão (40\%) ficou elevado devido principalmente à variação encontrada no primeiro trecho de pilar, mas esta pode ser desconsiderada devido à sua pouca influência no dimensionamento. Nas expressões de interação, a variação média foi de apenas $2,3 \%$, com um desvio-padrão de $1 \%$.

Tabela 3 - Comparação entre as solicitações de cálculo do vento com efeitos de vizinhança e uniforme. (Fonte: Camargo, 2012)

\begin{tabular}{|c|c|c|c|c|c|c|c|c|c|c|c|c|}
\hline \multirow{2}{*}{ Pilar } & \multicolumn{3}{|c|}{ Compressão } & \multicolumn{3}{|c|}{ Flexão em X } & \multicolumn{3}{|c|}{ Flexão em Y } & \multicolumn{3}{|c|}{ Expr. de interação } \\
\hline & Viz. & Unif. & Variação & Viz. & Unif. & Variação & Viz. & Unif. & Variação & Viz. & Unif. & Variação \\
\hline P1 & 0,36 & 0,35 & $2,9 \%$ & 0,10 & 0,10 & $0,0 \%$ & 0,04 & 0,02 & $100,0 \%$ & 0,47 & 0,45 & $4,4 \%$ \\
\hline P2 & 0,46 & 0,46 & $0,0 \%$ & 0,10 & 0,09 & $11,1 \%$ & 0,04 & 0,03 & $33,3 \%$ & 0,58 & 0,57 & $1,8 \%$ \\
\hline P3 & 0,48 & 0,48 & $0,0 \%$ & 0,13 & 0,12 & $8,3 \%$ & 0,06 & 0,05 & $20,0 \%$ & 0,65 & 0,64 & $1,6 \%$ \\
\hline P4 & 0,40 & 0,40 & $0,0 \%$ & 0,13 & 0,13 & $0,0 \%$ & 0,07 & 0,07 & $0,0 \%$ & 0,58 & 0,57 & $1,8 \%$ \\
\hline P5 & 0,34 & 0,34 & $0,0 \%$ & 0,14 & 0,13 & $7,7 \%$ & 0,11 & 0,10 & $10,0 \%$ & 0,56 & 0,55 & $1,8 \%$ \\
\hline \multicolumn{3}{|c|}{ Média } & $0,6 \%$ & - & - & $5,4 \%$ & - & - & $32,7 \%$ & - & - & $2,3 \%$ \\
\hline \multicolumn{3}{|c|}{ Desvio padrão } & $1 \%$ & - & - & $5 \%$ & - & - & $40 \%$ & - & - & $1 \%$ \\
\hline
\end{tabular}

Ao comparar os esforços solicitantes dos pilares obtidos pelo método P-Delta com aqueles fornecidos pelo MAES (Tabela 4 e Tabela 5), observa-se que os esforços de compressão dos pilares P2 e P3 apresentaram valores contra a segurança, com desvio de $5,5 \%$ e $11,4 \%$, respectivamente. Os momentos fletores atuantes em $Y$ tiveram um desvio-padrão relativamente alto $(4,5 \%)$, visto que o maior e o menor desvio foram, respectivamente, 7,3\% e 3,0\%. Além disso, as variações das forças cortantes máximas nas duas direções foram desprezíveis.

No que se refere aos deslocamentos laterais e aos deslocamentos interpavimentos, os maiores valores encontrados foram $10 \mathrm{~cm}$ e $6,8 \mathrm{~mm}$, respectivamente, quando o vento é aplicado na direção $\mathrm{Y}$ e com os efeitos de vizinhança inseridos. Estes valores atendem aos limites $\mathrm{H} / 400(17,5 \mathrm{~cm})$ e $\mathrm{h} / 500(7 \mathrm{~mm})$.

Assim, é possível concluir que a estrutura utilizada no modelo 1 atende a todos os requisitos referentes ao seu dimensionamento. Seu consumo de aço ficou em cerca de $1.204,92$ ton. Desse total, 610,2 ton $(50,6 \%)$ são referentes às vigas e 594,72 ton $(49,4 \%)$ são devidas aos pilares. Considerando uma área total de $18.000 \mathrm{~m}^{2}$, a taxa global ficou igual a $66,94 \mathrm{~kg} / \mathrm{m}^{2}$. 
Tabela 4 - Comparação entre os esforços solicitantes obtidos pelo MAES e método PDelta, considerando o vento com efeitos de vizinhança. (Fonte: Camargo, 2012)

\begin{tabular}{|c|c|c|c|c|c|c|c|c|c|}
\hline \multirow{2}{*}{ Pilar } & \multicolumn{3}{|c|}{ Compressão (kN) } & \multicolumn{3}{|c|}{ Flexão em X (kNcm) } & \multicolumn{3}{|c|}{ Flexão em Y (kNcm) } \\
\hline & P-Delta & $B_{1}-B_{2}$ & Variação & P-Delta & $B_{1}-B_{2}$ & Variação & P-Delta & $B_{1}-B_{2}$ & Variação \\
\hline P1 & 9.358 & 9.304 & $0,6 \%$ & 104.186 & 102.282 & $1,9 \%$ & -6.516 & -6.221 & $4,7 \%$ \\
\hline P2 & 6.792 & 7.190 & $-5,5 \%$ & -53.144 & -54.818 & $-3,1 \%$ & -6.207 & -6.469 & $-4,1 \%$ \\
\hline P3 & 4.803 & 5.421 & $-11,4 \%$ & -44.897 & -45.921 & $-2,2 \%$ & -6.166 & -6.356 & $-3,0 \%$ \\
\hline P4 & 3.689 & 3.692 & $-0,1 \%$ & -32.385 & -32.951 & $-1,7 \%$ & -5.531 & -5.759 & $-4,0 \%$ \\
\hline P5 & 2.020 & 2.017 & $0,2 \%$ & -19.775 & -19.981 & $-1,0 \%$ & -3.804 & -4.106 & $-7,3 \%$ \\
\hline \multicolumn{3}{|c|}{ Média } & $-3,3 \%$ & - & - & $-1,2 \%$ & - & - & $-2,7 \%$ \\
\hline \multicolumn{3}{|c|}{ Desvio padrão } & $5,2 \%$ & - & - & $1,9 \%$ & - & - & $4,5 \%$ \\
\hline
\end{tabular}

Tabela 5 - Comparação entre os esforços solicitantes obtidos pelo MAES e método PDelta, considerando o vento com efeitos de vizinhança. (Fonte: Camargo, 2012)

\begin{tabular}{|c|c|c|c|c|c|c|}
\hline \multirow{2}{*}{ Pilar } & \multicolumn{3}{|c|}{ Cortante em X (kN) } & \multicolumn{3}{|c|}{ Cortante em Y (kN) } \\
\hline & P-Delta & $B_{1}-B_{2}$ & Variação & P-Delta & $B_{1}-B_{2}$ & Variação \\
\hline P1 & 98 & 97 & $1,3 \%$ & 383 & 379 & $1,2 \%$ \\
\hline $\mathrm{P} 2$ & 99 & 97 & $1,9 \%$ & 335 & 331 & $1,1 \%$ \\
\hline P3 & 104 & 102 & $1,7 \%$ & 267 & 265 & $0,6 \%$ \\
\hline P4 & 106 & 105 & $1,1 \%$ & 185 & 191 & $-3,3 \%$ \\
\hline P5 & 82 & 84 & $-2,9 \%$ & 115 & 116 & $-0,5 \%$ \\
\hline \multicolumn{3}{|c|}{ Média } & $0,6 \%$ & - & - & $-0,2 \%$ \\
\hline \multicolumn{3}{|c|}{ Desvio padrão } & $2,0 \%$ & - & - & $1,9 \%$ \\
\hline
\end{tabular}

\subsection{Modelo 2}

O modelo 2 consiste em um sistema estrutural composto por pórticos contraventados em $X$ nos eixos 1 e 6 , entre as filas $B$ e $C$, e em $K$ nas filas $A$ e $D$, entre os eixos 1 e 2, 5 e 6, com bases engastadas. Os pórticos dos eixos 2, 3, 4 e 5 foram mantidos. As vigas V5 e V2 das regiões contraventadas, e as barras das diagonais de contraventamento foram consideradas como rotuladas nas extremidades. As hipóteses adotadas para a incidência do vento e para o dimensionamento de vigas e pilares foram as mesmas apresentadas para o modelo 1, sendo que no caso dos pilares foi adotada a redução da sobrecarga de utilização ao longo da altura da estrutura.

O perfil utilizado nas diagonais foi o HP $250 \times 62$. As vigas mistas e pilares de aço obtidos estão mostrados na Tabela 6 e Tabela 7. Percebe-se que, com a introdução de contraventamentos, não ocorreram alterações nas seções das vigas em relação àquelas utilizadas no modelo 1 , mas os pilares apresentaram pesos e alturas menores. Além disso, houve um acréscimo de peso devido às diagonais. 
Tabela 6 - Características das vigas mistas. (Fonte: Camargo, 2012)

\begin{tabular}{|c|c|c|c|c|c|}
\hline \multirow{2}{*}{ Viga } & \multirow{2}{*}{ Seção } & \multicolumn{4}{|c|}{ Perfil de aço } \\
\hline & & $\mathrm{d}(\mathrm{mm})$ & $b_{f}(\mathrm{~mm})$ & $t_{f}(\mathrm{~mm})$ & $t_{w}(\mathrm{~mm})$ \\
\hline V1 & VS $500 \times 61$ & 500 & 250 & 9,5 & 6,3 \\
\hline V2 & VS $500 \times 61$ & 500 & 250 & 9,5 & 6,3 \\
\hline V3A & VS $500 \times 61$ & 500 & 250 & 9,5 & 6,3 \\
\hline V3B & VS $500 \times 61$ & 500 & 250 & 9,5 & 6,3 \\
\hline V4 & VS $750 \times 108$ & 750 & 320 & 12,5 & 8,0 \\
\hline V5 & VS $750 \times 108$ & 750 & 320 & 12,5 & 8,0 \\
\hline V6 & VS $750 \times 108$ & 750 & 320 & 12,5 & 8,0 \\
\hline V7 & VS $750 \times 108$ & 750 & 320 & 12,5 & 8,0 \\
\hline
\end{tabular}

Tabela 7 - Características dos pilares de aço. (Fonte: Camargo, 2012)

\begin{tabular}{|c|c|c|c|c|c|c|}
\hline \multirow{2}{*}{ Pilar } & \multirow{2}{*}{ Andares } & \multirow{2}{*}{ Seção } & \multicolumn{4}{|c|}{ Perfil de aço } \\
\hline & & & $\mathrm{d}(\mathrm{mm})$ & $b_{f}(m m)$ & $t_{f}(\mathrm{~mm})$ & $t_{w}(\mathrm{~mm})$ \\
\hline P1 & $1^{\circ}-4^{\circ}$ & CVS $600 \times 278$ & 600 & 400 & 31,5 & 19 \\
\hline P2 & $5^{\circ}-8^{\circ}$ & CVS $600 \times 278$ & 600 & 400 & 31,5 & 19 \\
\hline P3 & $9^{\circ}-12^{\circ}$ & CVS $600 \times 190$ & 600 & 400 & 19 & 16 \\
\hline P4 & $13^{\circ}-16^{\circ}$ & PS 600 x 144 & 600 & 350 & 16 & 12,5 \\
\hline P5 & $17^{\circ}-20^{\circ}$ & PS $600 \times 112$ & 600 & 300 & 12,5 & 9,5 \\
\hline
\end{tabular}

Após o processo de classificação da estrutura, observou-se que a utilização dos contraventamentos teve pouca influência na deslocabilidade. Considerando os deslocamentos dos nós superiores dos pilares, fator $R_{s}$ igual a 1,00 para os pórticos com contraventamentos e igual a 0,85 para os sem contraventamentos, os maiores valores do coeficiente $B_{2}$ ficaram iguais a 1,10 (com redução da sobrecarga) e 1,12 (sem redução da sobrecarga). Como esperado, esses valores foram encontrados nas combinações com maiores cargas gravitacionais (combinação sem a ação do vento e naquela em que a sobrecarga é a ação variável principal). Assim, a estrutura mantevese com uma deslocabilidade média.

Foi realizada a classificação da estrutura dividindo-se os deslocamentos de segunda ordem $\left(u_{2}\right)$, obtidos pelo método P-Delta, pelos deslocamentos de primeira ordem $\left(u_{1}\right)$. O maior valor obtido para $\mathrm{u}_{2} / \mathrm{u}_{1}$ foi 1,07 , quando a redução da sobrecarga de utilização não é adotada. Nesse caso, a estrutura seria de pequena deslocabilidade.

Para o dimensionamento do edifício, os coeficientes $B_{2}$ foram recalculados simplificadamente com o módulo de elasticidade reduzido. Mais uma vez, o máximo valor foi obtido para o eixo 3 e a combinação que forneceu este valor foi aquela em que a atuação do vento é desprezada e há apenas forças nocionais. Quando é assumida a redução da sobrecarga ao longo da altura da estrutura, esse valor ficou igual a 1,12. Quando essa premissa não é adotada, o coeficiente $B_{2}$ passou para 1,14 
(Figura 4). Em todos os casos, os coeficientes $B_{1}$ ficaram limitados ao seu valor mínimo, ou seja, iguais a 1,00 .

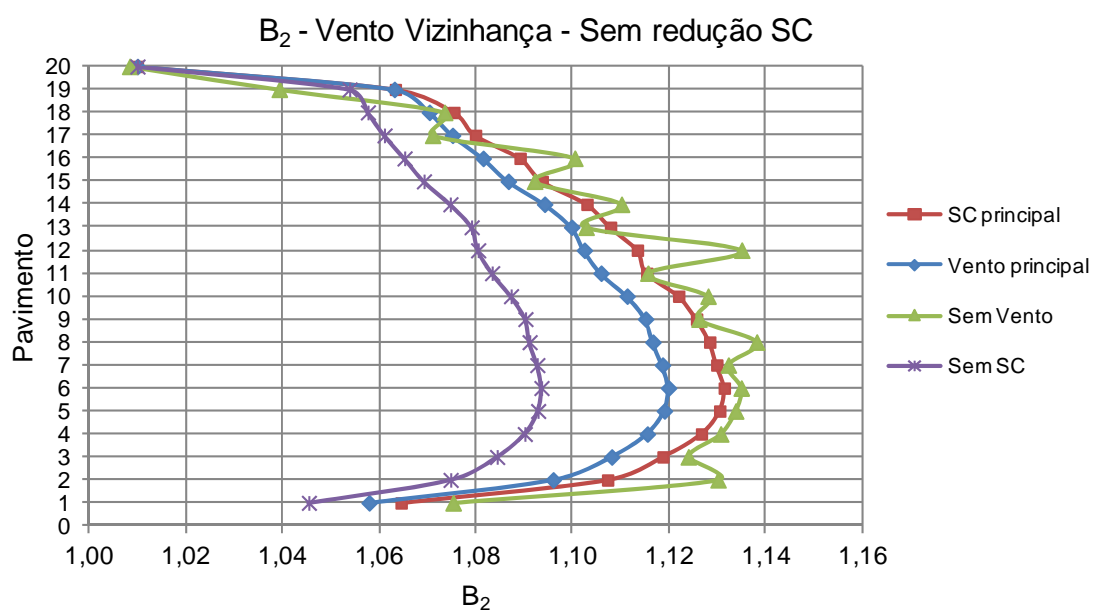

Figura 4 - Variação do coeficiente $B_{2}$, por pavimento, considerando o vento com efeitos de vizinhança em $Y$, quando aplicável, e sem redução da sobrecarga - Eixo 3.

(Fonte: Camargo, 2012)

Analisando os esforços encontrados para o vento uniforme, percebe-se uma grande semelhança com os valores obtidos para o vento com os efeitos de vizinhança. Como pode ser visto na Tabela 8, as maiores diferenças ocorreram nos momentos fletores. As relações entre as forças de compressão solicitantes e resistentes de cálculo apresentaram uma significativa variação para o primeiro trecho de pilares. Isso ocorreu devido ao fato de o pilar mais solicitado para cada caso de incidência do vento pertencer a filas diferentes.

No caso dos contraventamentos, as variações entre os esforços solicitantes foram consideráveis (Tabela 9). Porém, essa diferença era prevista, em virtude dos acréscimos de deslocamentos provocados pelos efeitos de vizinhança.

Quando os esforços solicitantes dos pilares fornecidos pelo método P-Delta são comparados àqueles fornecidos pelo MAES (Tabela 10 e Tabela 11), percebe-se que as variações são desprezíveis para os três tipos de solicitações. No entanto, a força normal do pilar mais solicitado do segundo trecho, obtida pelo método P-Delta, ficou cerca de 5,3\% menor, elevando o desvio-padrão dos esforços de compressão. Para os contraventamentos (Tabela 12), o esforço solicitante máximo obtido pelo método PDelta ficou maior para o caso de compressão e menor para a tração. 
Tabela 8 - Comparação entre as solicitações de cálculo dos pilares, considerando vento com efeitos de vizinhança e uniforme. (Fonte: Camargo, 2012)

\begin{tabular}{|c|c|c|c|c|c|c|c|c|c|c|c|c|}
\hline \multirow{2}{*}{ Pilar } & \multicolumn{3}{|c|}{ Compressão } & \multicolumn{3}{|c|}{ Flexão em X } & \multicolumn{3}{|c|}{ Flexão em Y } & \multicolumn{3}{|c|}{ Expr. de interação } \\
\hline & Viz. & Unif. & Variação & Viz. & Unif. & Variação & Viz. & Unif. & Variação & Viz. & Unif. & Variação \\
\hline P1 & 0,80 & 0,79 & $1,3 \%$ & 0,15 & 0,14 & $7,1 \%$ & 0,02 & 0,01 & $100,0 \%$ & 0,95 & 0,92 & $3,3 \%$ \\
\hline P2 & 0,59 & 0,59 & $0,0 \%$ & 0,16 & 0,16 & $0,0 \%$ & 0,06 & 0,05 & $20,0 \%$ & 0,78 & 0,77 & $1,3 \%$ \\
\hline P3 & 0,66 & 0,66 & $0,0 \%$ & 0,22 & 0,21 & $4,8 \%$ & 0,10 & 0,09 & $11,1 \%$ & 0,94 & 0,93 & $1,1 \%$ \\
\hline P4 & 0,66 & 0,66 & $0,0 \%$ & 0,21 & 0,20 & $5,0 \%$ & 0,12 & 0,11 & $9,1 \%$ & 0,95 & 0,94 & $1,1 \%$ \\
\hline P5 & 0,52 & 0,52 & $0,0 \%$ & 0,22 & 0,21 & $4,8 \%$ & 0,20 & 0,19 & $5,3 \%$ & 0,88 & 0,87 & $1,1 \%$ \\
\hline \multicolumn{3}{|c|}{ Média } & $0,3 \%$ & - & - & $4,3 \%$ & - & - & $29,1 \%$ & - & - & $1,6 \%$ \\
\hline \multicolumn{3}{|c|}{ Desvio padrão } & $1 \%$ & - & - & $3 \%$ & - & - & $40 \%$ & - & - & $1 \%$ \\
\hline
\end{tabular}

Tabela 9 - Comparação entre as solicitações de cálculo dos contraventamentos, considerando vento com efeitos de vizinhança e uniforme. (Fonte: Camargo, 2012)

\begin{tabular}{|c|c|c|c|c|c|c|}
\hline \multirow{2}{*}{ Perfil } & \multicolumn{2}{|c|}{ Compressão (kN) } & \multirow{2}{*}{ Variação } & \multicolumn{2}{|c|}{ Tração (kN) } & \multirow{2}{*}{ Variação } \\
\hline & Vizinhança & Uniforme & & Vizinhança & Uniforme & \\
\hline HP $250 \times 62$ & 1082 & 916 & $18,1 \%$ & 420 & 252 & $66,7 \%$ \\
\hline
\end{tabular}

Tabela 10 - Comparação entre os esforços solicitantes obtidos pelo MAES e método P-

Delta, considerando o vento com efeitos de vizinhança. (Fonte: Camargo, 2012)

\begin{tabular}{|c|c|c|c|c|c|c|c|c|c|}
\hline \multirow{2}{*}{ Pilar } & \multicolumn{3}{|c|}{ Compressão (kN) } & \multicolumn{3}{|c|}{ Flexão em X (kNcm) } & \multicolumn{3}{|c|}{ Flexão em Y (kNcm) } \\
\hline & P-Delta & $B_{1}-B_{2}$ & Variação & P-Delta & $B_{1}-B_{2}$ & Variação & P-Delta & $B_{1}-B_{2}$ & Variação \\
\hline P1 & 8.224 & 8.221 & $0,0 \%$ & 38.699 & 40.325 & $-4,0 \%$ & -1.242 & -1.238 & $0,4 \%$ \\
\hline P2 & 5.696 & 6.016 & $-5,3 \%$ & 43.341 & 44.204 & $-2,0 \%$ & -4.446 & -4.479 & $-0,7 \%$ \\
\hline P3 & 4.453 & 4.582 & $-2,8 \%$ & -36.879 & -37.645 & $-2,0 \%$ & -4.438 & -4.498 & $-1,3 \%$ \\
\hline P4 & 3.155 & 3.170 & $-0,5 \%$ & -26.865 & -27.338 & $-1,7 \%$ & -3.195 & -3.313 & $-3,6 \%$ \\
\hline P5 & 1.780 & 1.784 & $-0,2 \%$ & -18.759 & -18.880 & $-0,6 \%$ & -3.193 & -3.228 & $-1,1 \%$ \\
\hline \multicolumn{3}{|c|}{ Média } & $-1,8 \%$ & - & - & $-2,1 \%$ & - & - & $-1,3 \%$ \\
\hline \multicolumn{3}{|c|}{ Desvio padrão } & $2,3 \%$ & - & - & $1,2 \%$ & - & - & $1,4 \%$ \\
\hline
\end{tabular}

Tabela 11 - Comparação entre os esforços solicitantes obtidos pelo MAES e método P-

Delta, considerando o vento com efeitos de vizinhança. (Fonte: Camargo, 2012)

\begin{tabular}{|c|c|c|c|c|c|c|}
\hline \multirow{2}{*}{ Pilar } & \multicolumn{3}{|c|}{ Cortante em X (kN) } & \multicolumn{3}{|c|}{ Cortante em Y (kN) } \\
\hline & P-Delta & $\mathrm{B}_{1}-\mathrm{B}_{2}$ & Variação & P-Delta & $B_{1}-B_{2}$ & Variação \\
\hline P1 & 60 & 60 & $0,0 \%$ & 236 & 238 & $-0,7 \%$ \\
\hline P2 & 69 & 69 & $0,5 \%$ & 234 & 235 & $-0,5 \%$ \\
\hline P3 & 75 & 75 & $0,4 \%$ & 193 & 197 & $-2,2 \%$ \\
\hline P4 & 55 & 55 & $0,3 \%$ & 164 & 169 & $-3,0 \%$ \\
\hline P5 & 53 & 53 & $-0,2 \%$ & 170 & 171 & $-0,6 \%$ \\
\hline \multicolumn{3}{|c|}{ Média } & $0,2 \%$ & - & - & $-1,4 \%$ \\
\hline \multicolumn{3}{|c|}{ Desvio padrão } & $0,3 \%$ & - & - & $1,1 \%$ \\
\hline
\end{tabular}

Tabela 12 - Solicitações de cálculo máximas dos contraventamentos, obtidas pelo método P-Delta e pelo MAES. (Fonte: Camargo, 2012)

\begin{tabular}{ccccccc}
\hline \multirow{2}{*}{ Perfil } & \multicolumn{3}{c}{ Compressão (kN) } & \multicolumn{3}{c}{ Tração $(\mathbf{k N})$} \\
\cline { 2 - 7 } & $\mathbf{P}-$ Delta & $\mathbf{B}_{\mathbf{1}}-\mathbf{B}_{\mathbf{2}}$ & Variação & P-Delta & $\mathbf{B}_{\mathbf{1}}-\mathbf{B}_{\mathbf{2}}$ & Variação \\
\hline P1 & 1.153 & 1.082 & $6,6 \%$ & 277 & 420 & $-34,0 \%$ \\
\hline
\end{tabular}

No que diz respeito aos deslocamentos interpavimentos, os maiores valores obtidos nas direções $Y$ e $X$ foram 7,0 e 6,2 mm, respectivamente, e estes atenderam ao limite de $7 \mathrm{~mm}$ (h/500). Para os deslocamentos laterais, o máximo valor encontrado quando 
o vento é aplicado de forma excêntrica ficou igual a $10,5 \mathrm{~cm}$ na direção $Y$ e $3,0 \mathrm{~cm}$ na direção X. Em ambos os casos, o limite de $H / 400(17,5 \mathrm{~cm})$ foi respeitado.

Assim, conclui-se que a estrutura utilizada no modelo 2 também atende a todos os requisitos referentes ao seu dimensionamento. Seu consumo total de aço ficou igual a aproximadamente 995 ton, sendo 588,6 ton (59\%) referentes às vigas, 336,7 ton (34\%) devidas aos pilares e 69,5 ton (7\%) correspondentes ao peso dos contraventamentos. Considerando a área total do edifício, a taxa global ficou igual a 55,27 kg/m².

\subsection{Modelo 3}

O modelo 3 é composto por pórticos contraventados em $X$ nos eixos 1, 2, 5 e 6, entre filas B e C; em K nas filas A e D, entre eixos 1 e 2, 5 e 6 . Nos eixos 3 e 4, e nas filas B e C, foram mantidos os pórticos sem contraventamentos. As vigas V2, V5 e V7 das regiões contraventadas e os contraventamento foram rotulados nas extremidades. Os pilares foram dimensionados com redução da sobrecarga de utilização.

As características das seções de vigas e pilares utilizadas no modelo 3 estão mostradas nas tabelas a seguir. Para as diagonais de contraventamento, foram utilizados os perfis W $250 \times 52$ e HP $250 \times 62$.

Tabela 13 - Características das vigas mistas. (Fonte: Camargo, 2012)

\begin{tabular}{|c|c|c|c|c|c|}
\hline \multirow{2}{*}{ Viga } & \multirow{2}{*}{ Seção } & \multicolumn{4}{|c|}{ Perfil de aço } \\
\hline & & $\mathrm{d}(\mathrm{mm})$ & $b_{f}(\mathrm{~mm})$ & $t_{f}(\mathrm{~mm})$ & $t_{w}(\mathrm{~mm})$ \\
\hline V1 & VS $500 \times 61$ & 500 & 250 & 9,5 & 6,3 \\
\hline V2 & VS $500 \times 61$ & 500 & 250 & 9,5 & 6,3 \\
\hline V3A & VS $500 \times 61$ & 500 & 250 & 9,5 & 6,3 \\
\hline V3B & VS $500 \times 61$ & 500 & 250 & 9,5 & 6,3 \\
\hline V4 & VS $700 \times 105$ & 700 & 320 & 12,5 & 8,0 \\
\hline V5 & VS $700 \times 105$ & 700 & 320 & 12,5 & 8,0 \\
\hline V6 & VS $700 \times 105$ & 700 & 320 & 12,5 & 8,0 \\
\hline V7 & VS $700 \times 105$ & 700 & 320 & 12,5 & 8,0 \\
\hline
\end{tabular}

Tabela 14 - Características dos pilares de aço. (Fonte: Camargo, 2012)

\begin{tabular}{ccccccc}
\hline \multirow{2}{*}{ Pilar } & \multirow{2}{*}{ Andares } & \multirow{2}{*}{ Seção } & $\mathbf{d}(\mathbf{m m})$ & $\mathbf{b}_{\mathbf{f}}(\mathbf{m m})$ & $\mathbf{t}_{\mathbf{f}}(\mathbf{m m})$ & $\mathbf{t}_{\mathbf{w}}(\mathbf{m m})$ \\
\hline P1 & $1^{\circ}-4^{\circ}$ & CVS $600 \times 278$ & 600 & 400 & 31,5 & 19 \\
P2 & $5^{\circ}-8^{\circ}$ & CVS $600 \times 226$ & 600 & 400 & 25 & 16 \\
P3 & $9^{\circ}-12^{\circ}$ & CVS $600 \times 190$ & 600 & 400 & 19 & 16 \\
P4 & $13^{\circ}-16^{\circ}$ & CVS 600 $\times 156$ & 600 & 400 & 16 & 12,5 \\
P5 & $17^{\circ}-20^{\circ}$ & PS 400 $\times 116$ & 600 & 350 & 12,5 & 9,5 \\
\hline
\end{tabular}

A classificação da estrutura utilizou os mesmos critérios apresentados no modelo 2 . Nesse caso, foram obtidos valores de $B_{2}$ iguais a 1,09 para o caso com redução da sobrecarga e 1,11 quando essa redução é desconsiderada. Realizando a classificação 
da estrutura por meio da relação entre os deslocamentos de segunda $\left(u_{2}\right)$ e de primeira ordem $\left(u_{1}\right)$, o maior valor encontrado foi 1,07. Em ambos os casos, como esperado, os maiores resultados foram obtidos nas combinações com o maior valor das ações gravitacionais.

Como a estrutura apresentou uma deslocabilidade média devido ao maior valor encontrado para o $B_{2}$, os módulos de elasticidade dos materiais foram reduzidos e os coeficientes $B_{2}$ foram recalculados para o dimensionamento dos elementos que formam o modelo 3. Os maiores valores foram encontrados no pórtico do eixo 3, para a combinação em que a atuação do vento é desprezada. Quando a redução da sobrecarga é tomada como uma hipótese de cálculo, esse valor ficou igual a 1,11. No entanto, quando essa premissa não é adotada, esse fator aumentou para 1,14.

Ao comparar os esforços dos pilares encontrados para o vento com efeitos de vizinhança com aqueles obtidos para o vento uniforme, observou-se novamente uma grande semelhança entre eles, especialmente para os esforços axiais de compressão. Percebeu-se também que a situação onde o vento atua uniformemente gera momentos fletores com magnitudes um pouco menores. Além disso, as expressões de interação obtidas para o vento com efeitos de vizinhança ficaram, na média, 2,0\% maiores do que os valores obtidos pelo vento uniforme.

Comparando os esforços solicitantes dos pilares fornecidos pelo método P-Delta com aqueles fornecidos pelo MAES (Tabela 15 e Tabela 16), percebeu-se que, no geral, as variações foram pequenas. Para os esforços de compressão e de flexão em X (eixo de maior inércia), o método P-Delta forneceu valores menores do que o MAES. Porém, para os esforços de flexão em $\mathrm{Y}$ e para as forças cortantes em $\mathrm{X}$ e $\mathrm{Y}$, os resultados ficaram um pouco maiores.

Nos contraventamentos (Tabela 17), os esforços solicitantes máximos de compressão obtidos pelo método P-Delta ficaram 10\% maiores para as diagonais dos eixos 1 e 6 , e $3,2 \%$ menores para as diagonais dos eixos 2 e 5 . No caso dos esforços de tração, os valores máximos apresentaram variações significativas contra a segurança. No entanto, esse tipo de esforço não foi determinante para o dimensionamento das barras, visto que as forças de compressão ficaram entre 3 e 6 vezes maiores do que as de tração. 
Tabela 15 - Comparação entre os esforços solicitantes obtidos pelo MAES e método PDelta, considerando o vento com efeitos de vizinhança. (Fonte: Camargo, 2012)

\begin{tabular}{|c|c|c|c|c|c|c|c|c|c|}
\hline \multirow{2}{*}{ Pilar } & \multicolumn{3}{|c|}{ Compressão (kN) } & \multicolumn{3}{|c|}{ Flexão em X (kNcm) } & \multicolumn{3}{|c|}{ Flexão em Y (kNcm) } \\
\hline & P-Delta & $B_{1}-B_{2}$ & Variação & P-Delta & $B_{1}-B_{2}$ & Variação & P-Delta & $B_{1}-B_{2}$ & Variação \\
\hline P1 & 8.042 & 8.098 & $-0,7 \%$ & 32.431 & 34.654 & $-6,4 \%$ & -1.243 & -1.175 & $5,8 \%$ \\
\hline P2 & 5.696 & 5.992 & $-4,9 \%$ & 35.256 & 37.338 & $-5,6 \%$ & -4.984 & -4.963 & $0,4 \%$ \\
\hline P3 & 4.424 & 4.562 & $-3,0 \%$ & -32.397 & -34.450 & $-6,0 \%$ & -5.024 & -4.944 & $1,6 \%$ \\
\hline P4 & 3.117 & 3.157 & $-1,3 \%$ & -25.837 & -27.129 & $-4,8 \%$ & -5.004 & -4.839 & $3,4 \%$ \\
\hline P5 & 1.762 & 1.772 & $-0,5 \%$ & -18.470 & -18.949 & $-2,5 \%$ & -3.688 & -3.528 & $4,5 \%$ \\
\hline \multicolumn{3}{|c|}{ Média } & $-2,1 \%$ & - & - & $-5,0 \%$ & - & - & $3,2 \%$ \\
\hline \multicolumn{3}{|c|}{ Desvio padrão } & $1,9 \%$ & - & - & $1,5 \%$ & - & - & $2,2 \%$ \\
\hline
\end{tabular}

Tabela 16 - Comparação entre os esforços solicitantes obtidos pelo MAES e método PDelta, considerando o vento com efeitos de vizinhança. (Fonte: Camargo, 2012)

\begin{tabular}{cccccccc}
\hline \multirow{2}{*}{ Pilar } & \multicolumn{3}{c}{ Cortante em X (kN) } & & \multicolumn{3}{c}{ Cortante em $\mathbf{Y}(\mathbf{k N})$} \\
\cline { 2 - 3 } \cline { 6 - 7 } & P-Delta & $\mathbf{B}_{\mathbf{1}}-\mathbf{B}_{\mathbf{2}}$ & Variação & & P-Delta & $\mathbf{B}_{\mathbf{1}}-\mathbf{B}_{\mathbf{2}}$ & Variação \\
\hline P1 & 57 & 56 & $1,8 \%$ & & 212 & 210 & $1,0 \%$ \\
P2 & 61 & 60 & $1,7 \%$ & & 211 & 210 & $0,5 \%$ \\
P3 & 60 & 59 & $1,7 \%$ & & 198 & 200 & $-1,0 \%$ \\
P4 & 63 & 62 & $1,6 \%$ & & 175 & 175 & $0,0 \%$ \\
P5 & 50 & 49 & $2,0 \%$ & & 169 & 169 & $0,0 \%$ \\
\hline \multicolumn{3}{c}{ Média } & $\mathbf{1 , 8 \%}$ & & - & - & $\mathbf{0 , 1 \%}$ \\
\hline \multicolumn{3}{c}{ Desvio padrão } & $\mathbf{0 , 2 \%}$ & - & - & $\mathbf{0 , 7 \%}$ \\
\hline
\end{tabular}

Tabela 17 - Solicitações de cálculo máximas dos contraventamento, obtidas pelo método P-Delta e pelo MAES. (Fonte: Camargo, 2012)

\begin{tabular}{ccccccc}
\hline \multirow{2}{*}{ Perfil } & \multicolumn{3}{c}{ Compressão (kN) } & \multicolumn{3}{c}{ Tração (kN) } \\
\cline { 2 - 7 } & P-Delta & $\mathbf{B}_{\mathbf{1}}-\mathbf{B}_{\mathbf{2}}$ & Variação & P-Delta & $\mathbf{B}_{\mathbf{1}}-\mathbf{B}_{\mathbf{2}}$ & Variação \\
\hline W 200 x 52 & 933 & 848 & $10,0 \%$ & 84 & 262 & $-67,9 \%$ \\
HP 250 x 62 & 1.061 & 1.096 & $-3,2 \%$ & 148 & 188 & $-21,3 \%$ \\
\hline
\end{tabular}

No que se refere aos deslocamentos laterais, o máximo valor encontrado foi $10,4 \mathrm{~cm}$, quando o vento é aplicado com excentricidade na direção Y. Para o caso em que o mesmo atua na direção $X$, o maior valor encontrado foi de apenas $3,1 \mathrm{~cm}$. Em relação aos deslocamentos interpavimentos, os maiores valores obtidos nas direções $\mathrm{Y}$ e $\mathrm{X}$ foram 6,9 e 6,6 mm, respectivamente. Em todos os casos, os limites $\mathrm{H} / 400(17,5 \mathrm{~cm})$ e $\mathrm{h} / 500(7 \mathrm{~mm})$ foram atendidos com folga.

Assim, o consumo total de aço da estrutura do modelo 3 ficou igual a aproximadamente $1.009,6$ ton, sendo que 581,4 ton (58\%) foram referentes às vigas, 323,2 ton (32\%) foram devidas aos pilares e 105,0 ton (10\%) foi o peso dos contraventamentos. A taxa global ficou igual a $56,09 \mathrm{~kg} / \mathrm{m}^{2}$. 


\section{Conclusões}

Dentre os sistemas estruturais estudados, o que apresentou a menor taxa de consumo de aço foi aquele utilizado no modelo 2, que totalizou, sem o peso das ligações, 55,27 $\mathrm{kg} / \mathrm{m}^{2}$, seguido pelo modelo $3\left(56,09 \mathrm{~kg} / \mathrm{m}^{2}\right)$ e pelo modelo $1\left(66,94 \mathrm{~kg} / \mathrm{m}^{2}\right)$. Embora tenha apresentado um consumo cerca de $2 \%$ superior ao do modelo 2 , o modelo 3 poderia ser considerado o mais adequado entre os três sistemas estruturais estudados, pois, devido ao maior número de contraventamentos, as ligações tornar-se-iam mais simples nessas regiões, diminuindo o custo total da obra.

Do ponto de vista de projeto, constatou-se que a possibilidade de se reduzir a sobrecarga para o dimensionamento de pilares proporciona uma economia de material, mas tem como consequência o aumento do trabalho, pois exige a utilização de diferentes combinações de cálculo para o dimensionamento de vigas e pilares. Percebeu-se também que o critério apresentado na ABNT NBR 6120:1980 abre espaço para diferentes interpretações, principalmente na forma de se avaliar o número de andares acima de um determinado pavimento e definir os coeficientes a serem aplicados em cada um deles. Sendo assim, seria interessante uma revisão desses coeficientes para que os mesmos fossem definidos conforme a utilização do piso.

No que diz respeito aos efeitos de vizinhança, observou-se que sua maior influência está no aumento dos momentos fletores e dos deslocamentos da estrutura.

Em relação à avaliação dos efeitos de segunda ordem pelo método da amplificação dos esforços solicitantes (MAES), constatou-se que, para efeitos de classificação, a combinação de cálculo crítica é aquela que possui o maior carregamento gravitacional. Entretanto, para o dimensionamento dos elementos, é necessário estudar outras hipóteses de cálculo, principalmente aquelas em que o vento é a ação variável principal, visto que essas situações são determinantes no cálculo de pilares e de vigas que fazem parte de pórticos.

Os resultados obtidos pelo método P-Delta mostraram-se bastante semelhantes àqueles calculados pelo MAES. Na maior parte dos casos, eles ficaram um pouco menores, com desvios desprezíveis. Por outro lado, as relações entre os 
deslocamentos de segunda $\left(u_{2}\right)$ e primeira ordem $\left(u_{1}\right)$ tiveram um comportamento diferente dos coeficientes $B_{2}$, com valores menores do que esses coeficientes.

Por fim, observou-se que o MAES é um método mais trabalhoso devido à necessidade de se modelar diferentes tipos de estruturas (contida e não contida lateralmente) para

a determinação dos esforços finais. Nesse aspecto, o emprego do método P-Delta presente no pacote comercial utilizado foi mais atrativo, pois não apresenta essa duplicidade de análises.

\section{Agradecimentos}

Ao Conselho Nacional de Pesquisa e Desenvolvimento Científico, CNPq, pelo apoio financeiro e ao Departamento de Engenharia de Estruturas da EESC-USP.

\section{Referências bibliográficas}

ASSOCIAÇÃO BRASILEIRA DE NORMAS TÉCNICAS. NBR 6120: Cargas para o cálculo de estruturas de edificações. Rio de Janeiro, 1980.

ASSOCIAÇÃO BRASILEIRA DE NORMAS TÉCNICAS. NBR 6123: Forças devidas ao vento em edificações. Rio de Janeiro, 1988.

ASSOCIAÇÃO BRASILEIRA DE NORMAS TÉCNICAS. NBR 8800: Projeto de estruturas de aço e estruturas mistas de aço e concreto de edifícios. Rio de Janeiro, 2008.

CAMARGO, R.E.M. Contribuição ao estudo da estabilidade de edifícios de andares múltiplos em aço. 2012. 312 p. Dissertação (Mestrado em Engenharia de Estruturas) - Escola de Engenharia de São Carlos, Universidade de São Paulo, São Carlos, 2012.

SÁLES, J.J. Estudo do projeto e da construção de edifícios de andares múltiplos com estruturas de aço. 1995. 257 p. Tese (Doutorado em Engenharia de Estruturas) - Escola de Engenharia de São Carlos, Universidade de São Paulo, São Carlos, 1995.

ZIEMIAN, R.D. Guide to stability design criteria for metal structures. 6th ed. New Jersey: John Wiley and Sons, 2010. 